\title{
ON THE BREAKING OF WATER WAVES ON A SLOPING BEACH OF ARBITRARY SHAPE*
}

\author{
By MORTON E. GURTIN (Carnegie-Mellon University)
}

Summary. Greenspan [1] considered water waves of finite amplitude on a beach of constant slope. He proved that: $\left(G_{1}\right)$ A wave of elevation with nonzero slope at the front propagating shoreward into quiescent water always breaks before the shore. ${ }^{\dagger}$ $\left(G_{2}\right)$ Under the same conditions a wave of depression never breaks.

In this note we do not assume that the beach has constant slope, but rather we allow the depth to be an arbitrary smooth function of position. We show that the appropriate generalizations of Greenspan's results are: $\left(G_{1}{ }^{\prime}\right)$ In the above circumstances a wave of elevation always breaks; in particular, it breaks at the shore when the amplitude is sufficiently small, otherwise it breaks before the shore. $\left(G_{2}{ }^{\prime}\right)$ A wave of depression breaks if and only if a certain integral (involving only the depth function) is finite, and it never breaks away from shore.

Analysis. The conservation equations for mass and momentum in the (nonlinear) theory of shallow water are (see Stoker [3]):

$$
\eta_{t}+\{v(\eta+h)\}_{x}=0, \quad v_{t}+v v_{x}+g \eta_{x}=0
$$

where $h(x)+\eta(x, t)$ is the depth of the water, $h(x)$ is the undisturbed depth ahead of the wave, $v(x, t)$ is the (particle) velocity, and $g$ is the gravitational acceleration. We assume, in what follows, that $h(x)$ is continuously differentiable.

We now consider a wave front which at time $t=0$ occupies the position $x=0$ and which is propagating in the direction of increasing $x$. We assume that across the front:

(a) $v$ and $\eta$ are continuous;

(b) the first and second derivatives of $v$ and $\eta$ suffer (at most )jump discontinuities. We assume further that the region ahead of the wave is quiescent, so that

(c) $v(x, t)=\eta(x, t)=0$ for $0 \leq t \leq \hat{t}(x)$,

where $\hat{t}(x)$ is the time at which the wave passes the point $x$. Note that conditions (a) and (b) assert that the front is an acceleration wave.

Given a function $f(x, t)$, we write

$$
f^{-}(x)=\lim _{t \downarrow t(x)} f(x, t)
$$

for the value of $f$ immediately behind the wave. Then (a) and (c) imply that

$$
v^{-}=\eta^{-}=0
$$

* Received December 6, 1973.

† Cf. Carrier and Greenspan [2], who established the existence of waves of elevation with zero slope at the front which do not break. 
while (a)-(c) and Maxwell's Theorem (see, e.g., Truesdell and Toupin [4], Sec. 175) yield the compatibility relations

$$
c\left(v_{x}\right)^{-}=-\left(v_{\iota}\right)^{-}, \quad c\left(\eta_{x}\right)^{-}=-\left(\eta_{\iota}\right)^{-},
$$

where

$$
c=\left(\frac{d \hat{t}}{d x}\right)^{-1}
$$

is the velocity of the front. If we evaluate (1) just behind the wave, we conclude, with the aid of (3), that

$$
\left(\eta_{t}\right)^{-}=-h\left(v_{x}\right)^{-}, \quad\left(v_{t}\right)^{-}=-g\left(\eta_{x}\right)^{-} .
$$

If we assume that $\left(\eta_{x}\right)^{-} \neq 0$, then (4) and (6) yield the following well-known formula for the velocity $c$ :

$$
c=(g h)^{1 / 2} .
$$

Our next step will be to establish an explicit expression for the amplitude

$$
a=a(x)=\left(\eta_{x}\right)^{-} .
$$

By (4), (6), and (8),

$$
\left(v_{t}\right)^{-}=-g a, \quad\left(v_{x}\right)^{-}=g a / c .
$$

If we differentiate $(1)_{1}$ with respect to $x$ and $(1)_{2}$ with respect to $t$, and evaluate the resulting relations immediately behind the wave, we find, using (3) and (7)-(9), that

$$
c^{2}\left(v_{x x}\right)^{-}-\left(v_{t}\right)^{-}+\frac{2 g^{2} h_{x}}{c} a+\frac{3 g^{2}}{c} a^{2}=0 .
$$

Next, by (2) and (5),

$$
\frac{d}{d x}\left(v_{x}\right)^{-}=\left(v_{x x}\right)^{-}+\frac{1}{c}\left(v_{x t}\right)^{-}, \quad \frac{d}{d x}\left(v_{t}\right)^{-}=\left(v_{t x}\right)^{-}+\frac{1}{c}\left(v_{t \imath}\right)^{-},
$$

so that

$$
c^{2} \frac{d}{d x}\left(v_{x}\right)^{-}-c \frac{d}{d x}\left(v_{t}\right)^{-}=c^{2}\left(v_{x x}\right)^{-}-\left(v_{\imath t}\right)^{-} .
$$

Eqs. (7) and (9)-(11) yield the following differential equation for $a$ :

$$
\frac{d a}{d x}+\frac{3 h_{x}}{4 h} a+\frac{3}{2 h} a^{2}=0 .
$$

To solve this equation one notes that the substitution $a=b^{-1}$ yields a linear differential equation for $b$. This equation is easily solved; the solution in terms of the amplitude $a$ is

$$
a(x)=\frac{\left(\frac{h_{0}}{h(x)}\right)^{3 / 4}}{\frac{1}{a_{0}}+I(x)},
$$

where $a_{0}=a(0)$ and $h_{0}=h(0)$ are the initial amplitude and the initial depth, and

$$
I(x)=\frac{3 h_{0}^{3 / 4}}{2} \int_{0}^{x} h^{-7 / 4} \text {. }
$$


Results. We now assume that the front is propagating up a sloping beach with shore at $x=l$, so that $h(x)>0$ for $0 \leq x<l$ and

$$
h(l)=0 .
$$

We use the following terminology: the front corresponds to a wave of elevation (respectively, depression) if $a_{0}<0$ (respectively, $a_{0}>0$ ); the wave breaks if $a(x)$ becomes infinite at some point $x$. It is clear from (14) and the assumption containing (15) that $I(l)=\infty$ is a distinct possibility; when this is the case we define $1 / I(l)=0$. We are now in a position to state our main result; in the statement of this theorem conditions (a)-(c) are tacitly assumed to hold.

$A$ wave of elevation always breaks. In particular, the wave breaks before the shore when $\left|a_{0}\right|>1 / I(l)$, at the shore when $\left|a_{0}\right| \leq 1 / I(l)$.

$A$ wave of depression propagating up a beach for which $I(l)<\infty$ always breaks, but at the shore. On the other hand, when $I(l)=\infty$ a wave of depression never breaks.

This theorem is an immediate consequence of (13) and (14). Indeed, by the assumption containing (15) the numerator in (13) is strictly positive on $[0, l)$ and tends to infinity as $x \rightarrow l$. The results for waves of elevation are consequences of this remark and the following facts: the denominator is strictly negative at $x=0$ and increases monotonically as $x$ increases; the denominator vanishes for $x<l$ if and only if $\left|a_{0}\right|>1 / I(l)$. On the other hand, for a wave of depression the denominator is strictly positive at $x=0$, increases as $x$ increases, and is finite on $[0, l)$. Thus $a(x)$ is finite for $0 \leq x<l$. If $I(l)<\infty$, then $a(x) \rightarrow \infty$ as $x \rightarrow l$ and the wave breaks at the shore. If $I(l)=\infty$ both the numerator and the denominator tend to infinity as $x \rightarrow l$, but an application of $L$ 'Hospital's rule shows that

$$
a(x) \rightarrow-\frac{1}{2} h_{x}(l) \text { as } x \rightarrow l,
$$

and the wave does not break. This completes the proof.

Assume now that near the shore $h$ behaves like $h_{0}(1-x / l)^{p}$; i.e.,

$$
\lim _{x \rightarrow l} \frac{h(x)}{h_{0}\left(1-\frac{x}{l}\right)^{p}}=1 \quad \text { (for some } p>0 \text { ). }
$$

Then the integral $I$, defined in (14), is convergent or divergent according as $p<\frac{4}{7}$ or $p \geq \frac{4}{7}$. Thus in the present circumstances the second portion of the theorem can be restated as follows: a wave of depression breaks if and only if the beach is sufficiently deep near shore.

In view of the above remarks, $I(l)=\infty$ when the beach has constant slope; thus as a corollary of our theorem we have Greenspan's [2] result: for a linear beach a wave of elevation always breaks before the shore, a wave of depression never breaks.

\section{REFERENCES}

[1] H. P. Greenspan, J. Fluid Mech. 4, 330 (1958)

[2] G. F. Carrier and H. P. Greenspan, J. Fluid Mech. 4, 97 (1958)

[3] J. J. Stoker, Comm. Pure Appl. Math. 1, 9 (1948)

[4] C. Truesdell and R. A. Toupin, The classical field theories, in Handbuch der Physik, Vol. III/1, Berlin: Springer-Verlag (1960) 